\section{Prioritise patients, ensure safety and keep costs down}

Now is a time for healthcare providers and health-tech companies to come together. More than ever we have a responsibility to work collaboratively to support the professionals working hard within the dental market and to support patients in any way that is possible. Key challenges are ahead of us: dealing with a backlog of patients efficiently, costs of PPE equipment, nervous patients, and keeping staff healthy.

Doctify's aim is to continuously promote trust and transparency in healthcare. Part of that ethos is to use technology to connect patients and dentists in an easily accessible and contactless way. Therefore, on top of displaying patient reviews to reduce patient anxiety, Doctify's team has worked tirelessly to launch a safe and secure video tool. This has now connected over 10,000 patients to their healthcare providers during the COVID crisis. Doctify knows you are all very busy so it has also put its team's resources at your disposal to help get your practice set up.

It is crucial to reduce all possible setbacks upon reopening and Doctify's tools will help you prioritise patients for face-to-face appointments, keep costs down, and ensure patient and staff safety.

For more information visit https:// doctors.doctify.co.uk/what-is-doctify-1/, call 02034110652 or email alex.thomas@ doctify.co.uk.

\title{
A game-changing air filtration and disinfection system
}

Aura Air UK has launched what it describes as 'the world's smartest, most aggressive, compact, anti-virus, combined air filtration and disinfection system for under $£ 500$ '. Its unique ' 6 stage' filter system, patented smart air quality algorithms, combined with high precision air quality sensors and $\mathrm{CO} \&$ Fire detection, built into one compact unit, make it a game changer in this, up until now, traditional market.

Clinical trials testing its effectiveness, specifically against the coronavirus, are currently underway in one of the top ten medical centres in the world.

The unit measures just $37.5 \times 37.5 \times 15$ $\mathrm{cm}$, is designed to hang on the ceiling above the patient to ensure the most efficient and optimum extraction of aerosol droplets. Despite its small size, the unit using the latest fan technology will purify a $60 \mathrm{~m}^{3}$ room $\left(25 \mathrm{~m}^{2}\right.$ area) passing that total air volume through the filter six times per hour.

Traditional air filters only use UV-C light to kill viruses and bacteria. Aura Air specifically targets viruses, bacteria, mould and fungus in a unique triple combination.

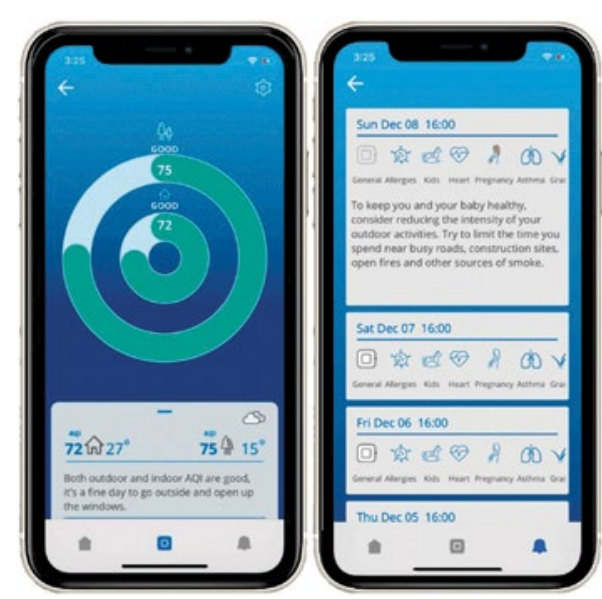

Firstly, the unique HEPA RAY Filter features a patented copper inlay. The patented copper impregnated HEPA filter releases ions when microbes land on the copper surface. The ions prevent cell respiration, punch holes in the bacterial cell membrane/disrupt the viral coat, and destroy the DNA and RNA inside.

This is backed up with a patented technology called a Sterionizer. This sends positive and negative oxygen ions into the air; the oxidants produced break down the protein structure of bacteria and viruses, fungus and mould not just in the air but on surfaces in the proximity of the filter. Other ionisers produce ozone which can damage the lungs of patients.

Finally, the third stage - traditional UVC LEDs - completes the disinfectant process.

The unit also features a carbon layer which absorbs VOCs and bad odours. The Class-13 level HEPA filter removes particulates down to 0.18 microns in size.

The Aura Air Filter system's inbuilt sensors accurately measure and display $\mathrm{Pm} 2.5$, Pm10 particulates levels as well as VOC, Humidity, $\mathrm{CO}$ and $\mathrm{CO} 2$ levels and compares these in real-time against the local outdoor conditions - all displayed in an easy to read manner on the app.

Aura Air has a patent pending on the algorithm controlling the air quality management system, customising the filter to each dentist's needs and health requirements. The device then detects relevant high risk scenarios and notifies the user through the Aura Air App.

For further information contact Aura Air UK on 02084204234 or email them atdpm@aura-air.co.uk. https://www. aurasmartair.co.uk/dentistry.html

\section{What challenge will you master today?}

Understanding that every patient is different, Straumann offers an all-inclusive range of biomaterials to help you overcome the daily challenges you face. The complete portfolio includes solutions to improve efficiency and effectiveness of bone grafting procedures, soft tissue grafts and periodontal surgery.

For example, Straumann Emodogain FL is designed to make periodontal regeneration less invasive. It can be used within flapless periodontal debridement procedures to efficiently and effectively reduce pockets, while optimising patient comfort at the same time.

Aside from its clinical benefits, Emodogain FL is a powerful tool in improving professional workflows and patient experiences.

Find out more about this and the many other innovative solutions within the Straumann biomaterials portfolio today.

For more information on the complete range of biomaterials, visit www. straumann-uk.co/biomaterials. 